between clinically assisted hydration and lower rates or delayed onset of delirium.

Conclusion There is insufficient evidence to draw conclusions on the impact of clinically assisted hydration on quality of life in the last days of life. Further research should focus on populations who are in the very final days of life. There is a lack of studies in non-cancer populations, and a lack of evidence relating to communication with patients and families about this uncertain area of clinical practice.

\section{IN YOUR OPINION WHAT ARE THE MOST IMPORTANT ETHICAL ISSUES FOR THE SPECIALTY IN THE NEXT FIVE YEARS AND HOW COULD WE ADDRESS THEM}

Beth Robbins. University of Leeds

10.1136/spcare-2020-PCC.17

A multitude of challenges are likely to confront the field of palliative care in the coming years, many of these with huge ethical implications. With demographic shifts resulting in the over-65 population resting at almost 12 million, combined with an unprecedented increase in the burden of diseases of old age such as dementia; it is likely that palliative care as a specialty will need to adapt to new demands. These challenges are compounded by considerable uncertainties surrounding funding, which will undoubtedly lead to difficult resource allocation decisions. Furthermore, ease of access to palliative care services varies considerably across society, with individuals with mental health conditions, a non-cancer diagnosis or belonging to BAME or LGBT communities all facing substantial barriers to access. This essay aims to examine how the fundamental principles of medical ethics such as beneficence, non-maleficence, justice and autonomy can be applied to the aforementioned challenges.

No proposals in isolation are likely to address all of the above challenges. Improved integration between general practice and palliative care services could significantly improve access to care however this will require further investment in an already overstretched primary care service. Extending opportunities to learn about palliative care to other health care professionals may also help to improve their confidence in treating this population. With projections indicating that care homes are likely to become the 'hospices of the future' it is vital that individuals working in these areas are adequately trained and able to work within a well-supported multi-disciplinary team. Disparities in access between societal groups will also need to be further studied in order for these to be levelled. Crucially addressing the above challenges must be done in a way that retains the holistic and individualistic nature, that is so integral to the speciality.

\section{ARE UK PALLIATIVE CARE PATIENTS WILLING TO PARTICIPATE IN A TRIAL OF BISPECTRAL INDEX (BIS) TECHNOLOGY FOR ASSESSING LEVELS OF CONSCIOUSNESS? FINDINGS FROM AN EXPLORATION OF FEASIBILITY FOR I-CAN-CARE}

Anna-Maria Krooupa, Paddy Stone, Stephen McKeever, Bella Vivat. University College London, Kingston University and St George's University London

10.1136/spcare-2020-PCC.18
Background Bispectral Index (BIS) monitoring uses electroencephalographic data to measure patients' level of consciousness. Our I-CAN-CARE study sought to explore the use of BIS in palliative care. Our preliminary work indicated that while a few clinicians had some reservations about the technology, patients and relatives did not. We therefore proceeded to investigate BIS in practice.

Objective To evaluate the feasibility of trialling BIS monitoring with conscious UK hospice patients.

Methods We conducted a prospective study of BIS with hospice inpatients, trialling the technology for a period of four hours. Feasibility was assessed against the following a priori criteria: percentage of recruited patients $15 \%$, and three criteria related to BIS specifically: percentage of eligible patients refusing to be approached for technology-related reasons $10 \%$; percentage of technology-related refusals to participate $10 \%$; and rate of non-completion due to the technology $10 \%$.

Results In 12 months, 332 hospice inpatients were screened for eligibility, and 177 (53.3\%) deemed eligible for the researcher to approach for consent. For reasons related to the technology, one patient refused approach (1/177); 34 could not be approached for other reasons. Six of those approached $(6 / 142 ; 4.2 \%)$ refused consent due to the technology. In all, $40 / 142(28.2 \%)$ agreed to participate. Thirty-nine of the 40 recruited were monitored with BIS (it was impossible to monitor the other patient successfully). Two participants (2/39; $5.1 \%$ ) requested that monitoring stop before the endpoint for reasons relating to the technology.

Conclusions Our findings show that it is feasible and acceptable to conduct research into BIS technology with conscious inpatients on a palliative care unit. The use of BIS did not markedly limit recruitment or retention, and most participants were content. Our analysis will proceed to examine data from these participants on the utility of BIS monitoring.

\section{Free papers 19 - 21: symptoms}

\section{MANAGEMENT OF OPIOID INDUCED HYPERALGESIA}

F Murray-Brown. Derriford Hospital, Plymouth

\subsection{6/spcare-2020-PCC.19}

Background Opioid-induced hyperalgesia (OIH) is a clinical phenomenon, characterised by increasing pain in patients who are receiving increasing doses of opioids. Many doctors are unaware of this phenomenon, often leading to a long latency between the classic presentation of the syndrome and its eventual recognition and treatment. There is no recognised management strategy for the treatment of $\mathrm{OIH}$ and several interventions have been used, with varying success.

Aim To review and assess the management of opioid-induced hyperalgesia in palliative patients.

Methods Electronic databases, grey literature, clinical trials registries and handsearching for studies describing OIH. Full papers were obtained if relevant and studies graded.

Results 30 papers were included in the analysis, including case reports and case series of a total of 54 patients. Clinical features included worsening or more widely distributed pain. $50 \%$ of patients also had myoclonus, $35 \%$ allodynia, and $15 \%$ agitation. 1 patient (2\%) suffered a seizure. Ten different interventions were used for the management of $\mathrm{OIH}$, with many patients receiving more than one intervention 